\title{
A Case Series of Conjunctival Rhinosporidiosis: Extremes of Presentation!!
}

\section{Shobita Nair ${ }^{1 *}$, Anitha Venugopal ${ }^{1}$, Vinit J Shah ${ }^{2}$ and Meenakshi Ravindran $^{1}$ \\ ${ }^{1}$ Department of Cornea, Aravind Eye Hospital, Tirunelveli, Tamil Nadu, India \\ ${ }^{2}$ Department of Retina Vitreous, Aravind Eye Hospital, Tirunelveli, Tamil Nadu, \\ India}

*Corresponding Author: Shobita Nair, Department of Cornea, Aravind Eye

Hospital, Tirunelveli, Tamil Nadu, India.
Received: July 16, 2020

Published: July 31, 2020

(C) All rights are reserved by Shobita Nair., et

al.

\begin{abstract}
Rhinosporidiosis is a rare infection caused by Rhinosporidium seeberi, an organism classified into class, Mesomycetozoea. It commonly affects mucus membranes. The diagnosis of rhinosporidiosis is based on its clinical appearance, microscopic features and the treatment is surgical excision. This condition is endemic in the temperate regions of the Indian subcontinent. Although a rare clinical entity, the possibility of rhinosporidiosis must be considered when evaluating any polypoidal conjunctival mass in patients belonging to any socioeconomic strata. Here we present 3 patients of different age groups and socioeconomic status with varied presentations suggestive of Conjunctival rhinosporidiosis, their evaluation, management, and outcomes.
\end{abstract}

Keywords: Rhinosporidiosis; Rhinosporidium seeberi; Conjunctiva; Staphyloma; Polypoidal Mass; Excision Biopsy

\section{Introduction}

Rhinosporidiosis is a chronic granulomatous infection caused by Rhinosporidium seeberi, a Mesomycetozoe endemic in parts of India and Sri Lanka. The nasal mucosa is most commonly affected but $10-15 \%$ of cases affect the conjunctiva and/or lacrimal sac $[1,2]$. The lesion presents as a polypoid mass characterized by friability, abundant vascularization, and pinpoint white spots on the surface $[3,4]$. Unusual presentations like scleral melting leading to staphyloma formation have also been reported [5]. Here we discuss 3 patients of different age groups with varied presentations coming from different socioeconomic strata diagnosed with Conjunctival rhinosporidiosis and their management.

\section{Materials and Methods}

Retrospective case series.

\section{Case Reports}

Case 1: A 10-Year-old male child, son of a farmer presented to our hospital with Right eye growth for 1 week with a history of taking bath in pond water 2 weeks back. Best-corrected visual acuity in both eyes was $6 / 12$ with myopic fundus. Right eye showed a lower tarsal conjunctival sessile mass $4 * 3 \mathrm{~mm}$ located centrally with overlying tiny white spots. The patient underwent Excision Biopsy with histopathological examination. Postoperatively the patient was put on antibiotic eye drops $0.5 \%$ Moxifloxacin 4 times a day for 2 weeks.

Case 2: A 22-Year-old female patient, engineering student presented to our hospital with Left eye redness and irritation with growth in her left eye increasing in size over 2 weeks. Visual acuity in both eyes was $6 / 6$. Anterior segment of the right eye was normal. Left eye showed a friable, polypoidal mass $5 * 3 \mathrm{~mm}$ with tiny white spots attached to the conjunctiva of the fornix centrally (Figure 1). Both eyes posterior segment was within normal limits. The patient underwent left eye excision biopsy and was put on $0.5 \%$ Moxifloxacin eye drops 4 times a day postoperatively for 2 weeks. The patient is on regular follow up without any evidence of recurrence. 


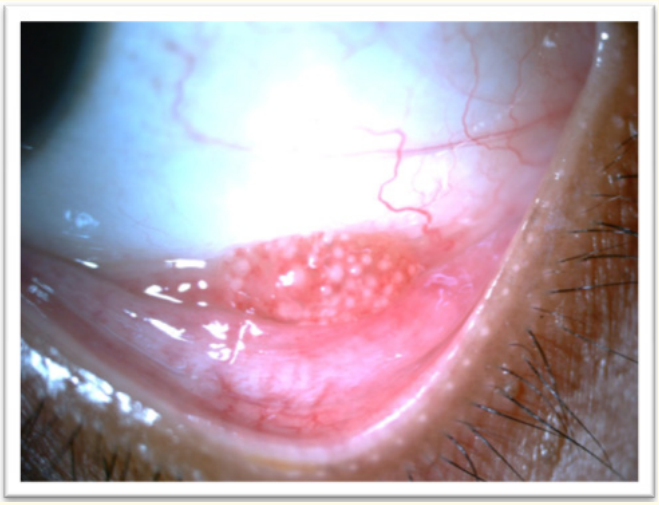

Figure 1: Slit lamp photograph of left eye showing a friable, polypoidal mass $5^{*} 3 \mathrm{~mm}$ with tiny white spots attached to the conjunctiva of the fornix suggestive of Conjunctival rhinosporidiosis.

Case 3: A 28-year-old female patient housewife by occupation presented to our hospital with black swelling in the Right eye for 3 years, as noticed by her husband. Visual acuity in both eyes was $6 / 6$. Anterior segment of the right eye showed an area of scleral ectasia measuring $10 * 10 * 6 \mathrm{~mm}$ over the supero-temporal part of the sclera and about $2 \mathrm{~mm}$ away from the limbus. The overlying conjunctiva showed dilated vessels and multiple small, discrete, yellowish-white polypoidal mass with mucoid spots on the surface (Figure 2). The left eye anterior segment was normal. Both eyes posterior segment was within normal limits. The mass was excised in toto, staphyloma was not disturbed and covered with Amniotic membrane graft with 8-0 vicryl suture. Postoperative 0.5\% Moxifloxacin and $0.5 \%$ Carboxymethyl cellulose drop 4 times a day was given for 2 weeks (Figure 3).

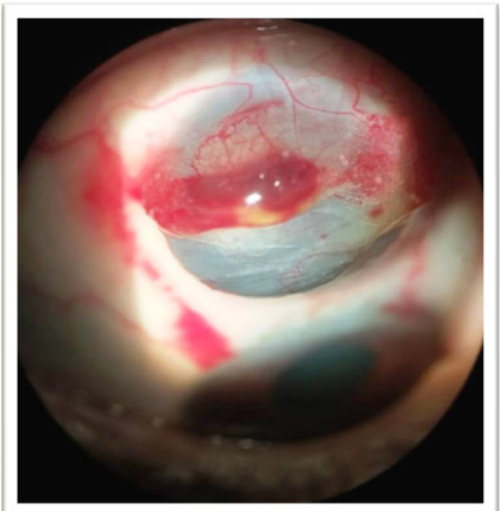

Figure 2: Preoperative slit lamp photograph of the right eye showing scleral ectasia measuring $10 * 10 * 6 \mathrm{~mm}$ over the superotemporal part of the sclera with overlying conjunctiva showing dilated vessels and multiple small, discrete, yellowish-white mucoid spots on the surface suggestive of Conjunctival rhinosporiodiosis.

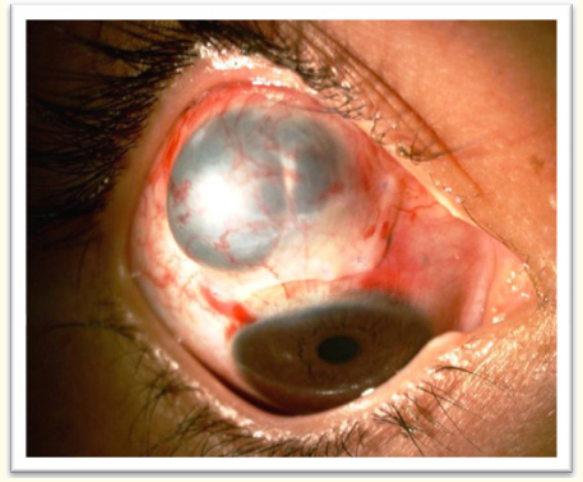

Figure 3: Postoperative photograph showing scleral staphyloma with Amniotic membrane in situ.

All 3 patients' excision biopsy report confirmed the diagnosis of Conjunctival Rhinosporidiosis (Figure 4). Histology shows multiple sporangia in different stages of maturity enclosed in a thin chitinous wall. The sporangia are $50-1000 \mu \mathrm{m}$ in diameter containing numerous endospores of 5 - $10 \mu \mathrm{m}$ diameters. The overlying epithelium is hyperplastic and loose fibrovascular stroma is infiltrated with lymphocytes, macrophages, plasma cells, and polymorphonuclear lymphocytes characteristic of Rhinosporidiosis.

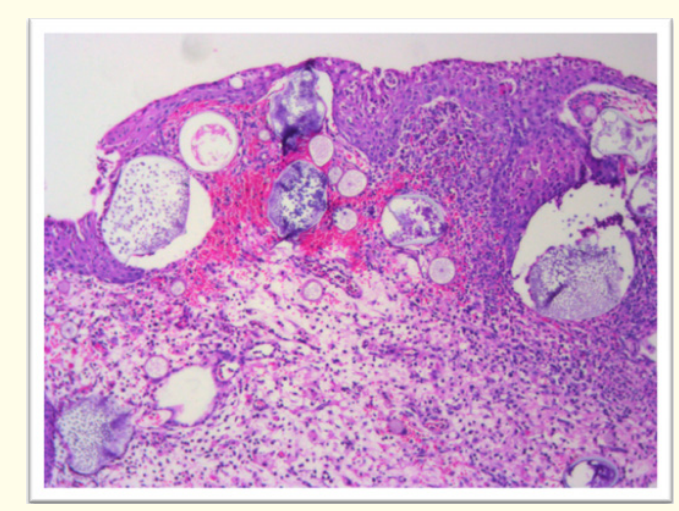

Figure 4: Multiple cystic lesions consisting of sporangia in various stages of degeneration are visible in the subepithelial stroma (H\&E Stain.10x). Mature spores are starting to protrude in the stroma. The thick-walled sporangia in SWISS CHEESE pattern are surrounded by a mononuclear inflammatory infiltrate (H\&E stain).

\section{Discussion and Conclusion}

Rhinosporidiosis is a chronic granulomatous and localized infection of the mucus membranes caused by Rhinosporidium seeberi. Although rhinosporidiosis occurs worldwide, it is endemic in Africa and Asia and is most frequent in India, Pakistan, and Sri Lanka 
(88\% of all reported cases) [1,4]. The first case was described as a nasal polyp-from Buenos Aires in 1900, by Guillermo Seeber. Herr., et al. classified this organism as Mesomycetozoa [2,8].

A male preponderance has been reported in most of the case reports with male: female ratio being 4.3:1 and higher prevalence in children and young adults between $3-39$ years $[4,10]$. Three patients described in this series are: 1 male child and 2 young females less than 30 years of age. Human infection is presumed to occur due to contact of the traumatized epithelium with contaminated groundwater and soil by infectious spore laden nasal discharge or feces of infected livestock or waterfowl $[3,4]$. A large population of poor villagers and people of semi-urban areas who are accustomed to taking bath in groundwater are prone to mucosal injuries $[2,8]$. In our series, only patient 1 gave the history of bathing in pond water while patients 2 and 3 were from higher socio-economic strata with no significant exposure history.

Nose and nasopharynx are the commonest sites involved but ocular lesions, particularly of the conjunctiva and lacrimal sac, account for $15 \%$ cases $[1,3]$. In our series also all were conjunctival lesions. Clinically, ocular rhinosporidiosis presents as a freely mobile, pink, friable, richly vascularized polypoid mass that is pedunculated or sessile. The surface is covered with tiny white spots consistent with underlying mature sporangia beneath the epithelium $[1,3]$. In our series, all patients had sessile lesions with all typical characteristics. Diagnosis is mainly clinical but confirmed by histopathology and meticulous excision is the treatment of choice $[6,10]$. Recurrences are rare and can be prevented by in toto excision followed by cauterization or cryotherapy at the base of the lesion. Similarly, in our series, patients 1 and 2 surgical excisions were done and no recurrences were noted over 2 years follow up. The potential for recurrence is due to the spillage of endospores on adjacent mucosa $[1,6]$.

Conjunctival rhinosporidiosis can cause scleral staphyloma in young, healthy adults leading to defective vision due to high astigmatism, scleral perforation, or secondary infection $[3,4]$. There have been only 13 cases of scleral melt and staphyloma formation reported in the literature to the best of our knowledge [9]. It has been postulated that some enzymatic substances produced by the organism corrode the sclera causing alterations in collagen resulting in weakening of the sclera, thinning, and staphyloma formation [6]. Rhinosporidiosis presenting as scleral staphyloma management is challenging and no specific guidelines have been men- tioned in the literature. Various reports have concluded that using a Scleral or corneal graft and covering the exposed sclera by either a Conjunctival flap or a properly secured Single or multiple layered Amniotic membranes is helpful. Indications for surgical intervention reported are defective vision due to high astigmatism and severe scleral thinning that can lead to scleral perforation $[6,7,10]$. In our case, first 2 patients had uncomplicated rhinosporidiosis and after excision, no recurrence was noted for 2 years while $3^{\text {rd }}$ patient with staphyloma; there was no indication for surgical intervention of the staphyloma so patient underwent excision of the conjunctival lesion alone and the exposed sclera was covered with amniotic membrane graft and patient was kept on regular follow up half-yearly.

Dapsone is the only drug that has had some success in treating Rhinosporidium seeberi but we did not use the drug in our series. Dapsone prevents the uptake of para-aminobenzoic acid by the microorganism by competitively inhibiting the enzyme, folate synthetase. This interferes with the synthesis of folic acid, which is essential for the formation of DNA. Thus, it decreases the formation and maturation of spores. It also causes fibrosis and shrinkage of the lesion, causing its regression and reducing the chances of recurrence $[1,3,6]$.

In conclusion, this case series emphasizes that Conjunctival rhinosporidiosis patients could be of different age groups and socioeconomic backgrounds even from nonendemic areas. They can have extremes of presentations from just a sessile conjunctival mass to a scleral staphyloma. Diagnosis requires a high index of clinical suspicion and familiarity with the conjunctival and scleral changes that will help in determining the surgical approach for treatment. When diagnosed early and with immediate intervention complications and recurrences can be prevented.

\section{Conflict of Interest}

The authors have no conflicts of interest to declare.

\section{Bibliography}

1. Kaimbo Wa Kaimbo., et al. "Conjunctival rhinosporidiosis: a case report from a Congolese patient". Bulletin de la Société belge d'ophtalmologie 309 (2008): 19-22.

2. Sinha A., et al. "Clinicopathological study of rhinosporidiosis with special reference to cytodiagnosis". Journal of Cytology 29.4 (2012): 246-249. 
3. Nair AG., et al. "Rhinosporidiosis of the tarsal conjunctiva". Indian Journal of Ophthalmology 63.5 (2015): 462-463.

4. Castelino AM, et al. "Conjunctival rhinosporidiosis associated with scleral melting and staphyloma formation: diagnosis and management". Cornea 19.1 (2000): 30-33.

5. De Doncker, R M et al. "Scleral melting in a patient with conjunctival rhinosporidiosis". The British Journal of Ophthalmology 74.10 (1990): 635-637.

6. John SS, et al. "Conjunctival oculosporidiosis with scleral thinning and staphyloma formation". Indian Journal of Ophthalmology 53.4 (2005): 272.

7. Jacob P., et al. "Tectonic corneal graft for conjunctival rhinosporidiosis with scleral melt". Indian journal of ophthalmology 59.3 (2011): 251.

8. Karthikeyan P., et al. "A retrospective epidemiological study of rhinosporidiosis in a rural tertiary care centre in Pondicherry". Journal of Clinical and Diagnostic Research: JCDR 10.5 (2015): MC04.

9. Luis Felipe da Silva Alves Carneiro., et al. "Case Report: Conjunctival Rhinosporidiosis Associated to Scleral Ectasia”. EC Ophthalmology 9.3 (2018): 135-139.

10. Costa EF., et al. "Partial regression of large anterior scleral staphyloma secondary to rhinosporidiosis after corneoscleral graft - a case report". BMC Ophthalmology 18.61 (2018).

\section{Assets from publication with us}

- Prompt Acknowledgement after receiving the article

- Thorough Double blinded peer review

- Rapid Publication

- Issue of Publication Certificate

- High visibility of your Published work

Website: www.actascientific.com/

Submit Article: www.actascientific.com/submission.php Email us: editor@actascientific.com

Contact us: +919182824667

Citation: Shobita Nair., et al. “A Case Series of Conjunctival Rhinosporidiosis: Extremes of Presentation!!”. Acta Scientific Ophthalmology 3.8 (2020): 57-60. 